\title{
Quantum Correlations of Helicity Entangled States in Non-inertial Frames Beyond Single Mode Approximation
}

\author{
Zeynab Harsi. * and Behrouz Mirzd \\ Department of Physics, Isfahan University of Technology, Isfahan 84156-83111, Iran
}

(Dated: May 25, 2021)

\begin{abstract}
A helicity entangled tripartite state is considered in which the degree of entanglement is preserved in non-inertial frames. It is shown that Quantum Entanglement remains observer independent. As another measure of quantum correlation, Quantum Discord has been investigated. It is explicitly shown that acceleration has no effect on the degree of quantum correlation for the bipartite and tripartite helicity entangled states. Geometric Quantum Discord as a Hilbert-Schmidt distance is computed for helicity entangled states. It is shown that living in non-inertial frames does not make any influence on this distance, either. In addition, the analysis has been extended beyond single mode approximation to show that acceleration does not have any impact on the quantum features in the limit beyond the single mode. As an interesting result, while the density matrix depends on the right and left Unruh modes, the Negativity as a measure of Quantum Entanglement remains constant. Also, Quantum Discord does not change beyond single mode approximation.
\end{abstract}

\section{INTRODUCTION}

Quantum correlations are fundamental tools in Quantum Information Theory [1]. They have found practical applications in recent discoveries in quantum cryptography [2, 3], quantum teleportation [4], quantum computer [5, 6], and quantum dense-coding [7]. Up until 2001, correlations not witnessed by Quantum Entanglement measures were thought not to be quantum correlations [8]. Henderson and Vedral 9], and Olivier and Zurek 10] introduced a new measure, called Quantum Discord, and concluded that Quantum Entanglement did not properly span all non-classical correlations. This measure has been quantitatively investigated recently [11, 12].

The study of quantum correlations in non-inertial frames plays an important role in investigating Quantum Information in black holes such as entropy and information paradox 13, 14. Adesso et al 15] and Mann et al [16] showed that entanglement of a scalar field in a non-inertial frame is affected by increasing acceleration. Alsing and Fuentes showed degradation of FermiDirac field entanglement 17]. However, the degradation of entanglement will occur from the perspective of a uniformly accelerated observer, which essentially originates from the fact that the event horizon appears and Unruh effect results in the loss of information for the non-inertial observer [18 20]. Moreover, Quantum Discord as a measure of quantum correlation is degraded in accelerated frames [21]. A bipartite helicity entangled state has been studied recently which has demonstrated that Quantum Entanglement of such a state remains unchanged while acceleration increases [22]. In this paper, a tripartite photon helicity entangled is considered where, the first, second, and third observers, respectively, represent an inertial observer,one, or two uniformly accelerated observers. What makes this interesting is the helicity entangled state taking the form of a mixed state, so that

\footnotetext{
* z.harsij@ph.iut.ac.ir

† b.mirza@cc.iut.ac.ir
}

the corresponding logarithmic negativity as a measure of Quantum Entanglement remains invariant against the acceleration of the second and third observers. This fact is completely different from the former entangled states in which the degradation of entanglement depended on the acceleration of the observer [20]. Quantum Entanglement does not include all quantum correlations; therefore, another measure should be computed to realize the acceleration dependence of this special correlation. In this paper, we considered these states and investigated their Quantum Discord as the difference between the two variant definitions of mutual information and showed that this quantum feature is also observer-independent.

All the observations and calculations have so far been limited to the single mode approximation, where observers only detect a single frequency mode 23]. But this approximation does not hold for all states. It is only appropriate for some special states which form some wave-packets by imposing fourier transformation 24]. It is shown that beyond the single-mode approximation, the Quantum Entanglement would depend on the type of wave-packets used in accelerated systems [25, [26]. Studying the relativistic quantum information beyond the single-mode approximation has been a recent topic of investigation [27, 28]. As another basic result, we show in this paper that this entangled state preserves its peculiarities in the limit beyond the single mode.

The rest of the paper is organized as follows: Section 2 gives an abstract view of states in an accelerated frame. In Section 3, Quantum Entanglement is studied and computed for the helicity tripartite entangled state in the non-inertial frame. Section 4 is devoted to the measure of quantum correlations. Quantum Discord will be computed for the specific bipartite and tripartite entangled states to investigate whether it is observer dependent or not. In Section 5, quantum correlations will be analyzed beyond the single mode approximation. Here, we realize that extending the single mode approximation would not make any difference for the acceleration dependence of these entangled states. Finally, the results and conclusions are presented in Section 6. 


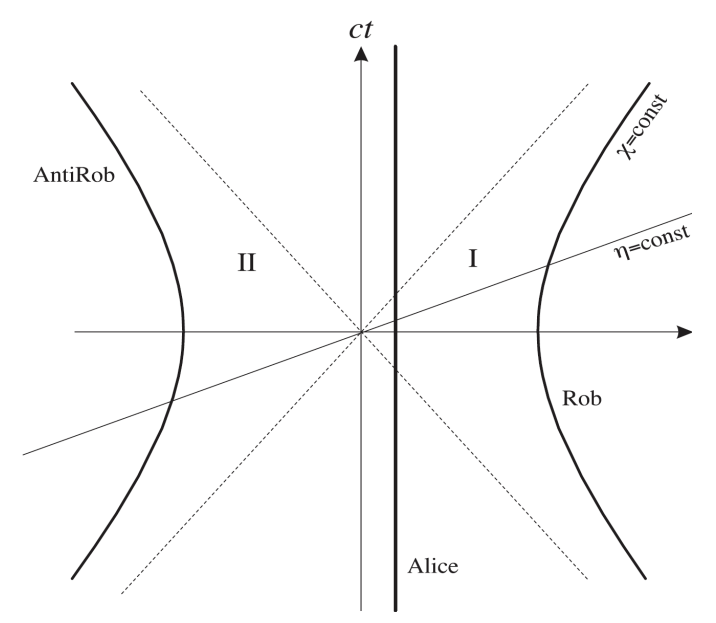

FIG. 1. Rindler space-time. As mentioned in the text, $\chi, \eta$ are Rindler coordinates and form the hyperbola. The two regions I, II are casually disconnected. Rob would travel in either one of these two regions.

\section{ACCELERATED FRAMES}

In investigating Quantum Entanglement and Quantum Discord in non-inertial frames, the observers should be on a hyperbola trajectory. Therefore, we use the Rindler coordinates to define the properties of the observers. These coordinates form four regions in a flat space-time. Here, we work with just two regions related to the Minkowski coordinates as follows

$$
\text { (I) }\left\{\begin{array}{c}
x=\frac{e^{a \chi}}{a_{a \chi}} \cosh a \eta \\
t=\frac{e}{a} \sinh a \eta
\end{array}\right\}, \quad(I I)\left\{\begin{array}{l}
x=\frac{-e^{a \chi}}{a_{a x}} \cosh a \eta \\
t=-\frac{e^{a}}{a} \sinh a \eta
\end{array}\right\}
$$

In this equation, $a$ represents the uniform acceleration of the non-inertial frame, $(x, t)$ are the Minkowski coordinates while $(\chi, \eta)$ are the Rindler coordinates. $(I)$ is the first Rindler region, which clearly differs from $(I I)$ by its sign while both are casually disconnected. The different signs indicate the two different directions in time. Since no information could flow from one region to another, we usually trace over one of them [17]. The two other Rindler regions are given by interchanging sinh by cosh. The Rindler regions are shown in Fig. 1.

Moreover, the concepts of particle and vacuum in non-inertial frames would change. Therefore, these are not basic concepts and therefore are observer-dependent. This effect, known as the Unruh effect, illustrates the fact that a vacuum in an inertial frame would make the non-inertial observer to feel a thermal bath of particles. In these frames the quantization is not unique and, as a consequence, the creation and annihilation operators would be in different forms which are related by the Bogolioubov coefficients [30]. Bogolioubov coefficients play important roles in defining observer dependent concepts.
In this paper, we are interested in investigating some of the quantum properties of entangled electromagnetic states. Consider the two observers Alice and Bob who are going to communicate and transfer information [19], where it is formal for Alice to be inertial and Bob to be in the accelerated frame. By this definition, Alice lives in Minkowski space-time whereas Bob is in the Rindler space-time. Thus, we will have

$$
|\psi\rangle=|A\rangle \otimes|B\rangle,
$$

where, $|A\rangle$ is formed for Alice and $|B\rangle$ for Bob in the accelerated frame. For Bob, the vacuum state in the Minkowski space-time could be defined as [19]

$$
|0\rangle^{M}=\left(1-e^{-2 \pi \omega}\right)^{\frac{1}{2}} \sum_{n=0}^{\infty} e^{-n \pi \omega}|n\rangle^{I}|n\rangle^{I I} .
$$

In this equation, we have assumed that the quantum states are bosonic. Acceleration in $\omega$ is designated by $\frac{E}{a}$ where $a$ denotes Bob's acceleration and $E$ is the energy which Bob detects. The other coefficients owe their presence to normalization and to the fact that $D|0\rangle^{M}=0$, where $D$ is the Minkowski annihilation operator which could be written in terms of creation and annihilation operators in Rindler ones:

$$
D=\frac{1}{\sqrt{ } 2 \sinh \omega}\left(e^{-\pi \omega} a^{I}-e^{\pi \omega} a^{\dagger I I}\right)
$$

$a^{I(I I)}, a^{\dagger I(I I)}$ are the first or the second Rindler annihilation and creation operators, respectively. For our purposes, the first excited state, $|1\rangle^{M}$, is also needed which could be easily derived by including $D^{\dagger}$ on the vacuum state in Eq. (3)

$$
\begin{aligned}
D^{\dagger}|0\rangle^{M} & =|1\rangle^{M} \\
& =\left(1-e^{-2 \pi \omega}\right) \sum_{n=0}^{\infty} e^{-2 n \pi \omega} \sqrt{(n+1)}|(n+1)\rangle^{I}|n\rangle^{I I} .
\end{aligned}
$$

In addition, since features of the helicity entangled states are to be investigated, they need to be introduced in the forms below [22]:

$$
\begin{aligned}
&|0\rangle_{\omega, p, s}^{M}=\left(1-e^{-2 \pi \omega}\right)^{\frac{1}{2}} \sum_{n=0}^{\infty} e^{-n \pi \omega}|n\rangle_{\omega, p, s}^{I}|n\rangle_{\omega,-p, s}^{I I}, \\
&|1\rangle_{\omega, p, s}^{M}=\left(1-e^{-2 \pi \omega}\right) \times \\
& \sum_{n=0}^{\infty} e^{-n \pi \omega} \sqrt{(n+1)}|(n+1)\rangle_{\omega, p, s}^{I}|n\rangle_{\omega,-p, s}^{I I} .
\end{aligned}
$$

In the above equations, $\omega$ indicates the energy impressing the observer, while $p$ and $s$ both show the helicity of the photon field. Using these definitions, the helicity entangled quantum properties can now be investigated.

\section{QUANTUM ENTANGLEMENT}

It has been shown that maximally entangled states have decrement in their degree of entanglement in noninertial frames [17, 19]. Degradation happens when acceleration is increased [20, 31]. With degradation, the information sent by Alice to other observers would be lost, 
which is not desirable. Therefore, these accelerated systems are not suitable for transmitting information. An entangled state which seems to send information without loss is a helicity entangled state which is like a WernerGHZ state with maximal entanglement but entangled in the helicity part. In defining this state, the helicity state which is moving with the uniform acceleration should be defined first. As an example, for a state which has a particle (photon) and a positive helicity we could have

$$
|1\rangle_{+\uparrow B}^{M}=\left(1-e^{-2 \pi \omega}\right) \sum_{n=0}^{\infty} e^{-n \pi \omega} \sqrt{(n+1)}|(n+1)\rangle_{+\uparrow}^{I}|n\rangle_{-\uparrow}^{I I},
$$

Other states are defined in a similar fashion. Here, the subindex $+(-)$ indicates the positive (negative) direction in momentum and $\mathrm{A}(\mathrm{B})$ is the Alice (Bob) observer. Also, $\uparrow(\downarrow)$ shows the spin up (down) of the photon. Helicity is defined as the multiple of spins and momentums of photon.

One measure of Quantum Entanglement (QE) is Negativity used to determine how far away two states are from separable states [32]. If it is equal to 1, then the state is maximally entangled; when it goes to zero, the state is separable; otherwise, it is partially entangled. In order to study the negativity, we need first to calculate the partial transpose of the density matrix which is constructed from the state given [33]. Peres presented a theorem which gives a necessary condition for separability. This theorem states that when the eigenvalues of the partial transpose of the Alice-Bob density matrix are positive, $\rho_{A B}^{P T} \geq 0$, then $A B$ is separable 34. As already mentioned, it is just a necessary condition and it does not guarantee the separability in systems other than the bipartite ones. If the above condition is satisfied, we could calculate the negativity defined as the sum of the negative eigenvalues of $\rho_{A B}^{P T}$ [32],

$$
\mathcal{N}=\log _{2} \sum_{i}\left|\lambda_{i}\right|
$$

in which a logarithmic negativity is introduced which is commonly used in bipartite systems. Also, we have summed over the absolute values of the eigenvalues, $\left|\lambda_{i}\right|$. Other definitions exist for negativity which could be used for systems greater than bipartite ones [35]

$$
\mathcal{N}=\left\|\rho^{P T}\right\|-1,
$$

where, $\rho^{P T}$ is the partial transpose of the density matrix in one of the subsystems of the multipartite system and $\|O\|$ denotes the trace norm of $O, \operatorname{Tr}\left[\sqrt{O^{\dagger} O}\right]$.

In order to investigate the frame dependence of $\mathrm{QE}$ for a general state, we study negativity of a tripartite system in which they are in a non-inertial frame and entangled in the helicity part. Thus, the state we would like to study is as follows

$$
|\psi\rangle=\frac{1}{\sqrt{2}}\left(|1\rangle_{+\uparrow A}^{M}|1\rangle_{-\downarrow B}^{M}|1\rangle_{-\downarrow C}^{M}+|1\rangle_{+\downarrow A}^{M}|1\rangle_{-\uparrow B}^{M}|1\rangle_{-\uparrow C}^{M}\right) .
$$

This state is similar to the Werner-GHZ one investigated in the reference [20], although both are maximally entangled; the difference, however, lies in their types of entanglement. In a tripartite system, we have three subsystems; namely, Alice is an inertial observer, Bob or Charlie, or both are the non-inertial observers. In tripartite systems, there are two entanglement measures: three-tangle and $\pi$-tangle [36]. The former is not of interest in this paper (simply because it cannot be computed analytically [37]). The latter, however, which is adapted as the quantification of entanglement in tripartite systems will be investigated here. The $\pi$-tangle is defined as the average of $\pi_{A}, \pi_{B}$, and $\pi_{C}$; that is:

$$
\pi=\frac{1}{3}\left(\pi_{A}+\pi_{B}+\pi_{C}\right) .
$$

Here, $\pi_{A}$ is defined as

$$
\pi_{A}=\mathcal{N}_{A(B C)}^{2}-\mathcal{N}_{A B}^{2}-\mathcal{N}_{A C}^{2},
$$

$\pi_{B}$ and $\pi_{C}$ are interpreted as the permutation of $\mathrm{A}$, $\mathrm{B}$, and C. $\mathcal{N}_{A B}$ is the negativity measure of the mixed state which is $\rho_{A B}=\operatorname{Tr}_{C}\left(|\psi\rangle_{A B C}\langle\psi|\right)$ and $\mathcal{N}_{A(B C)}$ is the one-tangle measure of negativity which is computed via Eq.(8). As will be shown in the following equations, this measure is computed when the density matrix is transposed in the inertial observer. This would define the entanglement between the inertial observer and the two accelerated ones.

For a system in which $\mathrm{A}$ is inertial, $\mathrm{B}$ and $\mathrm{C}$ are noninertial and when we have traced over the second Rindler region, the density matrix will be of the following form

$$
\begin{aligned}
& \rho_{A B_{I} C_{I}}=\operatorname{Tr}_{B_{I I}, C_{I I}}\left(\rho_{A B C}\right)=\frac{\left(1-e^{-2 \pi \omega_{B}}\right)^{2}}{2}\left(1-e^{-2 \pi \omega_{C}}\right)^{2} \times \\
& \sum_{n, m=0}^{\infty} e^{-2 n \pi \omega_{B}} e^{-2 m \pi \omega_{C}}(n+1)(m+1) \\
& \times \quad\left(| 1 \rangle _ { + \uparrow A } | n + 1 \rangle _ { - \downarrow B } | m + 1 \rangle _ { - \downarrow C } \left\langle1 | _ { + \uparrow A } \left\langlen+\left.1\right|_{-\downarrow B}\left\langle m+\left.1\right|_{-\downarrow C}\right.\right.\right.\right. \\
& +\quad|1\rangle_{+\uparrow A}|n+1\rangle_{-\downarrow B}|m+1\rangle_{-\downarrow C}\left\langle1 | _ { + \downarrow A } \left\langle n+\left.1\right|_{-\uparrow B}\left\langle m+\left.1\right|_{-\uparrow C}\right.\right.\right. \\
& +|1\rangle_{+\downarrow A}|n+1\rangle_{-\uparrow B}|m+1\rangle_{-\uparrow C}\left\langle1 | _ { + \uparrow A } \left\langle n+\left.1\right|_{-\downarrow B}\left\langle m+\left.1\right|_{-\downarrow C}\right.\right.\right. \\
& +|1\rangle_{+\downarrow A}|n+1\rangle_{-\uparrow B}|m+1\rangle_{-\uparrow C}\left\langle1 | _ { + \downarrow A } \left\langle n+\left.1\right|_{-\uparrow B}\left\langle m+\left.1\right|_{-\uparrow C}\right) .\right.\right.
\end{aligned}
$$


Here, subindex I means that we have traced over the second Rindler region in B and C. Also, $\omega_{B}\left(\omega_{C}\right)$ shows the B (C) acceleration frame. The partial transpose of this density matrix on the first observer, Alice, would take the following form

$$
\begin{aligned}
\rho_{A\left(B_{I} C_{I}\right)}^{P T} & =\frac{\left(1-e^{-2 \pi \omega_{B}}\right)^{2}}{2}\left(1-e^{-2 \pi \omega_{C}}\right)^{2} \\
\sum_{n, m=0}^{\infty} & e^{-2 n \pi \omega_{B}} e^{-2 m \pi \omega_{C}}(n+1)(m+1) \\
& \times \quad\left(| 1 \rangle _ { + \uparrow A } | n + 1 \rangle _ { - \downarrow B } | m + 1 \rangle _ { - \downarrow C } \left\langle1 | _ { + \uparrow A } \left\langlen+\left.1\right|_{-\downarrow B}\left\langle m+\left.1\right|_{-\downarrow C}\right.\right.\right.\right. \\
& +\quad|1\rangle_{+\downarrow A}|n+1\rangle_{-\downarrow B}|m+1\rangle_{-\downarrow C}\left\langle1 | _ { + \uparrow A } \left\langle n+\left.1\right|_{-\uparrow B}\left\langle m+\left.1\right|_{-\uparrow C}\right.\right.\right. \\
& +\quad|1\rangle_{+\uparrow A}|n+1\rangle_{-\uparrow B}|m+1\rangle_{-\uparrow C}\left\langle1 | _ { + \downarrow A } \left\langle n+\left.1\right|_{-\downarrow B}\left\langle m+\left.1\right|_{-\downarrow C}\right.\right.\right. \\
& +\quad|1\rangle_{+\downarrow A}|n+1\rangle_{-\uparrow B}|m+1\rangle_{-\uparrow C}\left\langle1 | _ { + \downarrow A } \left\langle n+\left.1\right|_{-\uparrow B}\left\langle m+\left.1\right|_{-\uparrow C}\right) .\right.\right.
\end{aligned}
$$

According to the definition presented in Eq. (8), $\mathcal{N}_{A\left(B_{I} C_{I}\right)}$, i.e. negativity, will be as follows:

$$
\begin{gathered}
\mathcal{N}_{A\left(B_{I} C_{I}\right)}=\frac{\left(1-e^{-2 \pi \omega_{B}}\right)^{2}}{2}\left(1-e^{-2 \pi \omega_{C}}\right)^{2} \\
\times\left(\sum_{n, m=0}^{\infty} e^{-2 n \pi \omega_{B}} e^{-2 m \pi \omega_{C}} 4(n+1)(m+1)\right)-1=1 .
\end{gathered}
$$

As already mentioned above, $\rho_{A B_{I}}$ is derived by tracing over the third subsystem $\mathrm{C}$

$$
\begin{aligned}
\rho_{A B_{I}} & =\operatorname{Tr}_{C}\left(\rho_{A\left(B_{I} C_{I}\right)}\right)=\frac{\left(1-e^{-2 \pi \omega_{B}}\right)^{2}}{2} \sum_{n=0}^{\infty} e^{-2 n \pi \omega_{B}} \\
& \times(n+1)\left(| 1 \rangle _ { + \uparrow A } | n + 1 \rangle _ { - \downarrow B } \left\langle1 | _ { + \uparrow A } \left\langlen+\left.1\right|_{-\downarrow B}\right.\right.\right. \\
& +|1\rangle_{+\downarrow A}|n+1\rangle_{-\uparrow B}\left\langle\left. 1\right|_{+\downarrow A}\left\langle n+\left.1\right|_{-\uparrow B}\right) .\right.
\end{aligned}
$$

Based on $|1\rangle_{+\uparrow A}|n+1\rangle_{-\uparrow B},|1\rangle_{+\uparrow A}|n+1\rangle_{-\downarrow B},|1\rangle_{+\downarrow A} \mid n+$ $1\rangle_{-\uparrow B},|1\rangle_{+\downarrow A}|n+1\rangle_{-\downarrow B}$, the matrix form of the density matrix $\rho_{A B_{I}}$ will be in the following form:

$\rho_{A B_{I}}=\frac{\left(1-e^{-2 \pi \omega_{B}}\right)^{2}}{2} \sum_{n=0}^{\infty} e^{-2 n \pi \omega_{B}}(n+1)\left(\begin{array}{cccc}0 & 0 & 0 & 0 \\ 0 & 1 & 0 & 0 \\ 0 & 0 & 1 & 0 \\ 0 & 0 & 0 & 0\end{array}\right)$

Clearly, this density matrix is in a diagonal form; therefore, by getting a partial transpose on either the A or $B$ part, it will not change and its eigenvalues will still be positive. Thus, as also mentioned in the Peres theorem, such a density matrix should not make an entangled state. Therefore, we will have $\mathcal{N}_{A B_{I}}=0 . \rho_{A C_{I}}$ could be derived in a similar manner and, as a consequence, $\mathcal{N}_{A C_{I}}=0$. Therefore, from Eq. (11), we will have

$$
\pi_{A}=1 \text {. }
$$

$\pi_{B}$ could be shown to be equal to $\pi_{C}$ which is itself equal to 1 . Now, we could derive $\pi$-tangle via Eq.(10) as follows:

$$
\pi=\frac{1}{3}\left(\mathcal{N}_{A(B C)}^{2}+\mathcal{N}_{B(A C)}^{2}+\mathcal{N}_{C(A B)}^{2}\right)=1 .
$$

It is evident that the tripartite system has preserved its degree of entanglement in a non-inertial frame. This particular helicity entangled state seems to have some other interesting consequences which are to be investigated in the next section.

\section{QUANTUM DISCORD}

QE introduced in the previous section is an evidence of quantum correlations, but it does not guarantee to include all quantum correlations. Although separable quantum states form a kind of quantum correlation, they are not included in QE measures. Quantum Discord (QD) is a more general evidence first presented by Olivier and Zurek [10]. This quantity defines the degree of quantum correlations and is defined as the difference between two expressions of mutual information in quantum while they are considered to be identical in classical terms [38]. In classical information theory, mutual information is the correlation between random variables and takes the following form for a bipartite system [10]

$$
\mathcal{J}(\mathcal{X}, \mathcal{Y})=\mathcal{H}(\mathcal{X})-\mathcal{H}(\mathcal{X} \mid \mathcal{Y})
$$

where, $\mathcal{H}$ is the Shanon entropy and is given by $\mathcal{H}=$ $-\sum \mathcal{P}(\mathcal{X}=x) \log \mathcal{P}(\mathcal{X}=x)$. Here, $\mathcal{P}(\mathcal{X})$ is the probability distribution for the random variable $\mathcal{X}$ to have the $x$-value. $\mathcal{H}(\mathcal{X} \mid \mathcal{Y})$ is the conditional entropy and may be written as:

$$
\mathcal{H}(\mathcal{X}, \mathcal{Y})-\mathcal{H}(\mathcal{Y})
$$

where, $\mathcal{H}(\mathcal{X}, \mathcal{Y})$ is the joint entropy; i.e., both $\mathcal{X}$ and $\mathcal{Y}$ occurring. Another expression for mutual information could be written in the following form 33]

$$
\mathcal{I}(\mathcal{X} ; \mathcal{Y})=\mathcal{H}(\mathcal{X})+\mathcal{H}(\mathcal{Y})-\mathcal{H}(\mathcal{X}, \mathcal{Y})
$$

It is evident that the two Eqs.(18) and (20) are equivalent in classical theory but they will behave differently when they are extended into the quantum systems. The difference would lie in the term QD. In the quantum version, $\mathcal{H}$ would explain the Von-Neumann entropy $\mathcal{S}$ which is defined in terms of density matrix as

$$
\mathcal{S}=-\operatorname{Tr}_{\mathcal{X}} \rho_{\mathcal{X}} \log _{2} \rho \mathcal{X} .
$$

Thus, for a bipartite system, Eq.(20) would take the form below:

$$
\begin{aligned}
\mathcal{I}(\mathcal{X} ; \mathcal{Y}) & =\mathcal{S}(\mathcal{X})+\mathcal{S}(\mathcal{Y})-\mathcal{S}(\mathcal{X}, \mathcal{Y}) \\
& =-\operatorname{Tr}\left(\rho_{\mathcal{X}} \log _{2} \rho_{\mathcal{X}}\right)-\operatorname{Tr}\left(\rho_{\mathcal{Y}} \log _{2} \rho_{\mathcal{Y}}\right) \\
& +\operatorname{Tr}\left(\rho_{\mathcal{X} \mathcal{Y}} \log _{2} \rho_{\mathcal{X Y}}\right)
\end{aligned}
$$


In addition, Eq. (18) would also change in the quantum system. Since the conditional entropy requires the state of $\mathcal{X}$ to be in a given state of $\mathrm{A}$, we need an optimized measurement approach [33]. This will be achieved by introducing some projection operators. Applying the optimized measurement approach would change Eq. (18) into the following form [21, 39]

$$
\mathcal{J}(\mathcal{X} ; A)=\mathcal{S}(\mathcal{X})-\min _{\pi_{i}}\left[\mathcal{S}\left(\rho_{\mathcal{X} \mid \pi_{i}^{A}}\right)\right]
$$

Evidently, the new expression for $\mathcal{J}$ differs from the former in their second term. This term is the optimized measurement of state $\mathcal{X}$ corresponding to $\pi_{i}^{A}$. The state is given as [40]

$$
\rho_{\mathcal{X} \mid \pi_{i}^{A}}=\frac{1}{P_{i}} \pi_{i}^{A} \rho_{\mathcal{X}, A} \pi_{i}^{A},
$$

where, $P_{i}$ is equal to $\operatorname{Tr}_{\mathcal{X}, A}\left(\pi_{i}^{A} \rho_{\mathcal{X}, A}\right)$. It is the probability for each measurement to have a given value. In the forthcoming subsection, we will present an explicit expression for QD in a bipartite system and will compute it for a particular case.

\section{IV.1. Quantum Discord for a bipartite helicity entangled state}

In the previous section, we investigated Quantum Entanglement for an entangled state. It was shown that this particular state does not behave as a usual entangled state does when it is observed in an accelerated frame. Similar to QE, QD is degraded by increasing acceleration [21]. Here, we would like to determine whether this specific case has similar features in QD. A density matrix which is commonly used for computing QD for a two-state system is as follows 33.

$$
\rho=\left(\frac{1-p}{4}\right) I+p|\psi\rangle\langle\psi|
$$

In this density matrix, $I$ is the identity and p is the probability given for finding a state in one of the states $|0\rangle$ or $|1\rangle$. The probability is, therefore, bounded, $0 \leq p \leq 1$. When $p=1, \rho$ is a pure state and when $p=0$, it is the identity. Using the following bipartite entangled state

$$
|\psi\rangle=\frac{1}{\sqrt{2}}\left(|1\rangle_{+\uparrow A}^{M}|1\rangle_{-\downarrow B}^{M}+|1\rangle_{+\downarrow A}^{M}|1\rangle_{-\uparrow B}^{M}\right),
$$

we can determine $\mathrm{QD}$ for a helicity entangled state. By putting the state given in Eq.(26) into Eq.(25) and by tracing over the second Rindler region for the Bob ob- server, we will clearly have the following density matrix

$$
\begin{aligned}
\rho_{A B_{I}} & =\left(1-e^{-2 \pi \omega}\right)^{2} \sum_{n=0}^{\infty} e^{-2 n \pi \omega}(n+1) \times \\
& \left(\frac { 1 - p } { 4 } \left(| 1 \rangle _ { + \uparrow A } | n + 1 \rangle _ { - \uparrow B } \left\langle1 | _ { + \uparrow A } \left\langlen+\left.1\right|_{-\uparrow B}\right.\right.\right.\right. \\
& +|1\rangle_{+\downarrow A}|n+1\rangle_{-\downarrow B}\left\langle\left. 1\right|_{+\downarrow A}\left\langle n+\left.1\right|_{-\downarrow B}\right)\right. \\
& +\frac{1+p}{4}\left(| 1 \rangle _ { + \uparrow A } | n + 1 \rangle _ { - \downarrow B } \left\langle1 | _ { + \uparrow A } \left\langlen+\left.1\right|_{-\downarrow B}\right.\right.\right. \\
& +|1\rangle_{+\downarrow A}|n+1\rangle_{-\uparrow B}\left\langle\left. 1\right|_{+\downarrow A}\left\langle n+\left.1\right|_{-\uparrow B}\right)\right. \\
& +\frac{p}{2}\left(|1\rangle_{+\uparrow A}|n+1\rangle_{-\downarrow B} \mid 1\left\langle_ { + \downarrow A } \left\langle n+\left.1\right|_{-\uparrow B}\right.\right.\right. \\
& +|1\rangle_{+\downarrow A}|n+1\rangle_{-\uparrow B}\left\langle\left. 1\right|_{+\uparrow A}\left\langle n+\left.1\right|_{-\downarrow B}\right)\right) .
\end{aligned}
$$

Based on $|1\rangle_{+\uparrow A}|n+1\rangle_{-\uparrow B}, \quad|1\rangle_{+\uparrow A}|n+1\rangle_{-\downarrow B},|1\rangle_{+\downarrow A} \mid n+$ $1\rangle_{-\uparrow B}, \quad|1\rangle_{+\downarrow A}|n+1\rangle_{-\downarrow B}, \rho_{A B_{I}}$ could be written in the matrix form as follows:

$$
\begin{aligned}
\rho_{A B_{I}} & =\left(1-e^{-2 \pi \omega}\right)^{2} \sum_{n=0}^{\infty} e^{-2 n \pi \omega}(n+1) \\
& \times\left(\begin{array}{cccc}
\frac{1-p}{4} & 0 & 0 & 0 \\
0 & \frac{1+p}{4} & \frac{p}{2} & 0 \\
0 & \frac{p}{2} & \frac{1+p}{4} & 0 \\
0 & 0 & 0 & \frac{1-p}{4}
\end{array}\right)
\end{aligned}
$$

which is an $\mathrm{X}$-shaped symmetric density matrix with real matrix elements, the difference lying in $\rho_{14}=\rho_{41}=0$. It is now possible to express QD more quantitatively. As mentioned before, QD, i.e. the difference between two mutual information pieces, takes the form below [10]:

$$
\begin{aligned}
\mathcal{D}(A: B) & =\mathcal{I}(A: B)-\mathcal{J}(A: B) \\
& =\mathcal{S}(A)+\mathcal{S}(B)-\mathcal{S}(A, B)-\mathcal{S}(A) \\
& +\min _{\left\{\pi_{i}^{A}\right\}} \mathcal{S}(A \mid B) .
\end{aligned}
$$

$\mathcal{S}(A), \mathcal{S}(B)$, and $\mathcal{S}(A, B)$ have been already explained above. The last term is the optimized, measured conditional entropy [10, 33]. This quantity has been presented for two qubit states in [40]. For a real symmetric X-state, $\mathcal{S}(A \mid B)$ over all projection operators is written as [39]:

$$
\mathcal{S}_{\pi_{i}}(A \mid B)=p_{0} \mathcal{S}\left(\rho_{A \mid 0}\right)+p_{1} \mathcal{S}\left(\rho_{A \mid 1}\right) .
$$

In two qubit X-states, we only have two p-probabilities, $p_{0}$ and $p_{1}$. Probabilities are related to each other by

$$
p_{0}=1-p_{1}=\left(\rho_{22}+\rho_{44}\right) l+\left(\rho_{11}+\rho_{33}\right) k .
$$

It should also be noticed that $\rho_{n m}=\langle n|\rho| m\rangle$. In addition, we have

$$
\mathcal{S}\left(\rho_{A \mid j}\right)=-\sum_{ \pm} \lambda_{ \pm}\left(\rho_{A \mid j}\right) \log _{2} \lambda_{ \pm}\left(\rho_{A \mid j}\right)
$$

where, $\lambda_{ \pm}\left(\rho_{A \mid j}\right)$ could be expressed as $\frac{1}{2}\left(1 \pm \theta_{j}\right)$, and $\theta_{j} \mathrm{~s}$ are

$$
\begin{aligned}
& \theta_{0}=\frac{1}{p_{0}} \sqrt{\left(\left(\rho_{11}-\rho_{33}\right) k+\left(\rho_{22}-\rho_{44}\right) l\right)^{2}+\beta} \\
& \theta_{1}=\frac{1}{p_{1}} \sqrt{\left(\left(\rho_{11}-\rho_{33}\right) l+\left(\rho_{22}-\rho_{44}\right) k\right)^{2}+\beta},
\end{aligned}
$$


and $\beta=4 k l\left(\rho_{14}+\rho_{23}\right)^{2}-16 \mu \rho_{14} \rho_{23}$. It is shown in 39. that the minimum value occurs when $k=l=\frac{1}{2}, \mu=0$ or $k=1-l=0,1$. According to the above equations, QD could be computed for any two qubit states

$$
\mathcal{D}(A \mid B)=\mathcal{S}(B)-\mathcal{S}(A, B)+\min _{\pi_{i}}[\mathcal{S}(A \mid B)]
$$

For the specific state defined in Eq. (26),

$$
\mathcal{S}(B)=-\sum \lambda_{\rho_{B}} \log _{2} \lambda_{\rho_{B}},
$$

where $\rho_{B}=\operatorname{Tr}_{A}\left(\rho_{A B_{I}}\right)=\frac{1}{2} I$, which is a multiple of identity. Thus, $\mathcal{S}(B)=1$. $\mathcal{S}(A, B)$ is defined in terms of the eigenvalues of $\rho_{A B_{I}}$. The eigenvalues are given by

$$
\begin{gathered}
\lambda_{1}=\lambda_{2}=\lambda_{3}=\left(1-e^{-2 \pi \omega}\right)^{2} \sum_{n=0}^{\infty} e^{-2 n \pi \omega}(n+1) \frac{1-p}{4}, \\
\lambda_{4}=\left(1-e^{-2 \pi \omega}\right)^{2} \sum_{n=0}^{\infty} e^{-2 n \pi \omega}(n+1) \frac{1+3 p}{4} .
\end{gathered}
$$

Therefore, $\mathcal{S}(A, B)$ is in the following form

$$
\begin{aligned}
\mathcal{S}(A, B) & =-\operatorname{Tr}\left(\rho_{A B} \log _{2} \rho_{A B}\right) \\
& =-3\left(\frac{1-p}{4}\right) \log _{2}\left(\frac{1-p}{4}\right)-\left(\frac{1+3 p}{4}\right) \log _{2}\left(\frac{1+3 p}{4}\right) .
\end{aligned}
$$

The last term which quantifies the QD could be computed from Eq. (32). For the particular state given in Eq. (26), $\lambda_{ \pm}=\frac{1}{2}\left(1 \pm \frac{p}{2}\right)$. Therefore, via Eq. (33), QD takes the following form

$$
\begin{aligned}
\mathcal{D}(A \mid B) & =1+3\left(\frac{1-p}{4}\right) \log _{2}\left(\frac{1-p}{4}\right)+\left(\frac{1+3 p}{4}\right) \log _{2}\left(\frac{1+3 p}{4}\right) \\
& -\frac{1}{2}\left(1+\frac{p}{2}\right) \log _{2} \frac{1}{2}\left(1+\frac{p}{2}\right)-\frac{1}{2}\left(1-\frac{p}{2}\right) \log _{2} \frac{1}{2}\left(1-\frac{p}{2}\right) \\
& =\frac{1}{4} \log _{2} \frac{(1+3 p)^{1+3 p}(1-p)^{1-p}}{(1+p)^{2(1+p)}} .
\end{aligned}
$$

Clearly, the quantity acceleration dependence disappears. We expect QD to be degraded by increasing acceleration whereas the above term does not depend on acceleration. Fig. 2 illustrates how QD depends on the p-parameter and is independent of acceleration. We could also study negativity for this density matrix using the same method introduced in Section [I] The result is shown in Fig. 3 It is observed that negativity for such a helicity entangled state is independent of acceleration, as it was in previous cases. It is interesting to note in Fig. 3 that if $p \geq \frac{1}{3}$, then an entangled state results.

\section{IV.2. Global Quantum Discord}

It is now desirable to extend the previous expression for QD to multipartite systems. For any arbitrary multipartite state, there is a global definition of QD which holds for any set of projection measurements $\left\{\pi_{i}\right\}$ [41]:

$$
\begin{aligned}
\mathcal{D}\left(\rho_{A_{1}} \ldots \rho_{A_{N}}\right) & =\min \left[\mathcal{S}\left(\rho_{A_{1} \ldots A_{N}} \| \phi\left(\rho_{A_{1} \ldots A_{N}}\right)\right)\right] \\
& -\sum_{j=1}^{N} \mathcal{S}\left(\rho_{A_{j}} \| \phi\left(\rho_{A_{j}}\right)\right),
\end{aligned}
$$

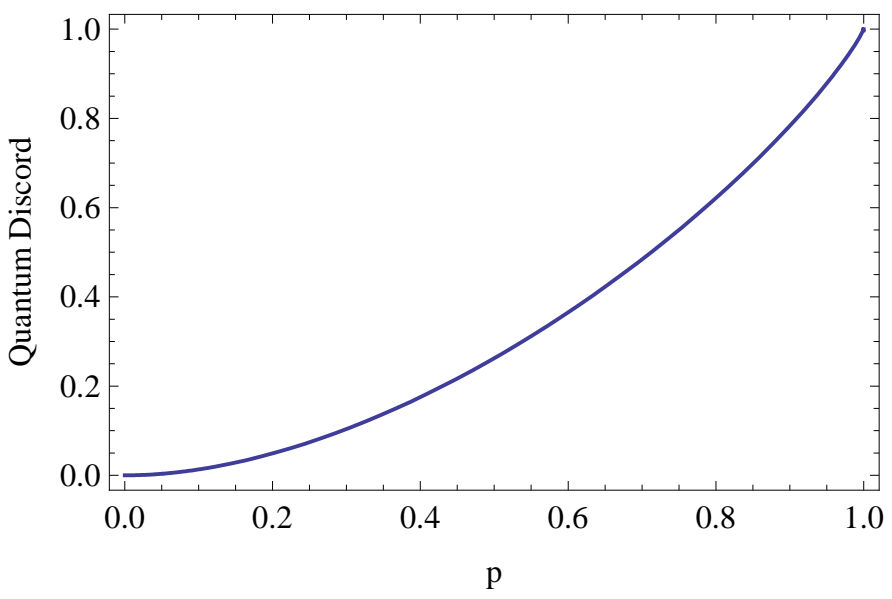

FIG. 2. Quantum Discord for a bipartite system versus p explicitly shows only p-dependence.

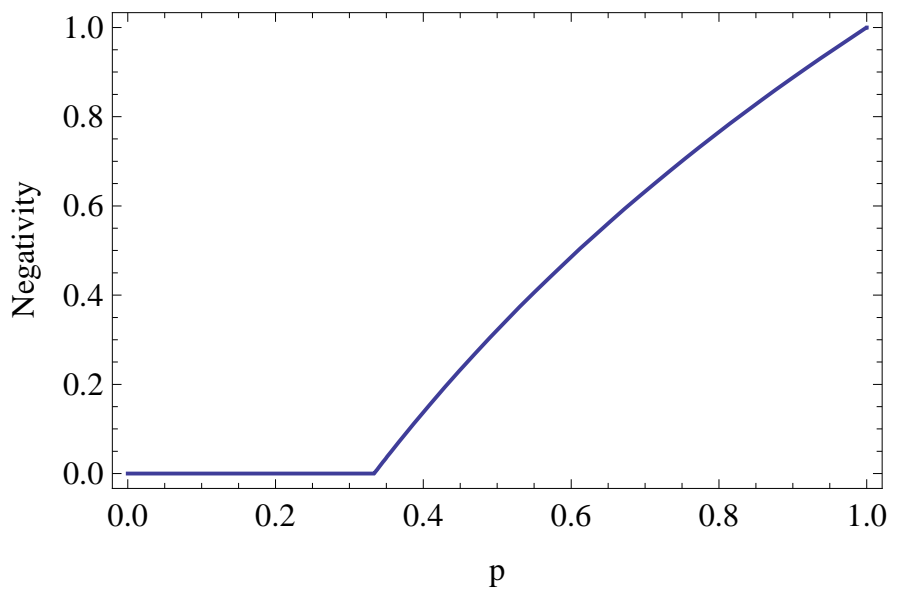

FIG. 3. Negativity versus p. For $p \geq \frac{1}{3}$, the state is entangled. No observer (acceleration) dependence is seen.

where, $\mathcal{S}(\rho \| \phi(\rho))=\mathcal{S}(\rho)-\mathcal{S}(\phi(\rho))$ is the relative entropy [33], $\phi_{j}\left(\rho_{A_{j}}\right)$ is equal to $\sum_{i} \pi_{A_{j}}^{i} \rho_{A_{j}} \pi_{A_{j}}^{i}$ and $\rho_{A_{j}}$ s are the density matrices for the different parts. Also, $\phi(\rho)$ could be found as $\sum_{k} \pi_{k} \rho_{A_{1} \ldots A_{N}} \pi_{k}$ with $\pi_{k}=\pi_{A_{1}}^{j_{1}} \otimes \ldots \otimes \pi_{A_{N}}^{j_{N}}$, which is a set of projection measurements made. This Global definition of QD satisfies the features of QD as stated in [41]. In addition to the generalization made for $\mathrm{QD}$, we would like to extend the density matrix given in Subsection IV.1 Eq. (25) to more than a bipartite state. For a multipartite system, the density matrix Eq.(25) would be extended as follows

$$
\rho=\frac{1-p}{2^{N}} I+p|\psi\rangle\langle\psi| .
$$

In the above expression, $I$ is a $2^{N}$ identity matrix and $\mathrm{p}$ is the probability as defined before. When $p \neq 0,1$, Eq. (36) provides a fully correlated state. It seems that the approach to be used here is different from the one used in the last subsection, but one could do the same calculations for $N=2$ to obtain similar results.

Let us now illustrate the global definition of QD with 
an example. The simplest example for a system higher than the bipartite one is a tripartite system, the density matrix for which is as follows:

$$
\rho_{A B C}=\frac{1-p}{8} I+p|\psi\rangle\langle\psi| .
$$

Here, $|\psi\rangle$ is exactly the state introduced in Eq.(9). Therefore, by tracing over the second regions, the density matrix would be of the following form

$$
\begin{aligned}
\rho_{A B_{I} C_{I}}= & \frac{\left(1-e^{-2 \pi \omega_{B}}\right)^{2}}{2}\left(1-e^{-2 \pi \omega_{C}}\right)^{2} \\
\sum_{n, m=0}^{\infty} & e^{-2 n \pi \omega_{B}} e^{-2 m \pi \omega_{C}}(n+1)(m+1)\left(\left(\frac{1-p}{8}\right) \times\right. \\
& \left(\quad | 1 \rangle _ { + \uparrow A } | n + 1 \rangle _ { - \uparrow B } | m + 1 \rangle _ { - \uparrow C } \left\langle1 | _ { + \uparrow A } \left\langlen+\left.1\right|_{-\uparrow B}\left\langle m+\left.1\right|_{-\uparrow C}\right.\right.\right.\right. \\
+ & |1\rangle_{+\uparrow A}|n+1\rangle_{-\uparrow B}|m+1\rangle_{-\downarrow C}\left\langle1 | _ { + \uparrow A } \left\langle n+\left.1\right|_{-\uparrow B}\left\langle m+\left.1\right|_{-\downarrow C}\right.\right.\right. \\
+ & |1\rangle_{+\uparrow A}|n+1\rangle_{-\downarrow B}|m+1\rangle_{-\uparrow C}\left\langle1 | _ { + \uparrow A } \left\langle n+\left.1\right|_{-\downarrow B}\left\langle m+\left.1\right|_{-\uparrow C}\right.\right.\right. \\
+ & |1\rangle_{+\downarrow A}|n+1\rangle_{-\uparrow B}|m+1\rangle_{-\downarrow C}\left\langle1 | _ { + \downarrow A } \left\langle n+\left.1\right|_{-\uparrow B}\left\langle m+\left.1\right|_{-\downarrow C}\right.\right.\right. \\
+ & |1\rangle_{+\downarrow A}|n+1\rangle_{-\downarrow B}|m+1\rangle_{-\uparrow C}\left\langle1 | _ { + \downarrow A } \left\langle n+\left.1\right|_{-\downarrow B}\left\langle m+\left.1\right|_{-\uparrow C}\right.\right.\right. \\
+ & |1\rangle_{+\downarrow A}|n+1\rangle_{-\downarrow B}|m+1\rangle_{-\uparrow C}\left\langle1 | _ { + \downarrow A } \left\langle n+\left.1\right|_{-\downarrow B}\left\langle m+\left.1\right|_{-\downarrow C}\right)\right.\right. \\
+\left(\frac{1+3 p}{8}\right) & \left(| 1 \rangle _ { + \uparrow A } | n + 1 \rangle _ { - \downarrow B } | m + 1 \rangle _ { - \downarrow C } \left\langle1 | _ { + \uparrow A } \left\langlen+\left.1\right|_{-\downarrow B}\left\langle m+\left.1\right|_{-\downarrow C}\right.\right.\right.\right. \\
+ & |1\rangle_{+\downarrow A}|n+1\rangle_{-\uparrow B}|m+1\rangle_{-\uparrow C}\left\langle1 | _ { + \downarrow A } \left\langle n+\left.1\right|_{-\uparrow B}\left\langle m+\left.1\right|_{-\uparrow C}\right)\right.\right. \\
+\quad & \left(| 1 \rangle _ { + \uparrow A } | n + 1 \rangle _ { - \downarrow B } | m + 1 \rangle _ { - \downarrow C } \left\langle1 | _ { + \downarrow A } \left\langlen+\left.1\right|_{-\uparrow B}\left\langle m+\left.1\right|_{-\uparrow C}\right.\right.\right.\right. \\
\quad & |1\rangle_{+\downarrow A}|n+1\rangle_{-\uparrow B}|m+1\rangle_{-\uparrow C}\left\langle\left. 1\right|_{+\uparrow A}\left\langle n+\left.1\right|_{-\downarrow B}\left\langle m+\left.1\right|_{-\downarrow C}\right)\right) .\right.
\end{aligned}
$$

Prior to the computation process, the measurements need to be defined first. If rotations are considered as projection operators, then in the directions of the basis vectors of $A, B$, and $C$ they may be defined as

$$
\begin{aligned}
& |+\rangle_{j}=\cos \left(\frac{\theta_{j}}{2}\right)|\uparrow\rangle_{j}+e^{\imath \phi_{j}} \sin \left(\frac{\theta_{j}}{2}\right)|\downarrow\rangle_{j}, \\
& |-\rangle_{j}=-e^{-\imath \phi_{j}} \sin \left(\frac{\theta_{j}}{2}\right)|\uparrow\rangle_{j}+\cos \left(\frac{\theta_{j}}{2}\right)|\downarrow\rangle_{j},
\end{aligned}
$$

where $j=A, B, C$. It should be noted that $\theta_{j} \epsilon[0, \pi)$, $\phi_{j} \epsilon[0,2 \pi)$ and the projection operators are found to be $\pi_{A_{j}}=| \pm\rangle\langle \pm|$. Using the global definition, Eq.(37), and inserting $N=3$, we get the following equation for QD in tripartite states

$$
\begin{aligned}
\mathcal{D}\left(\rho_{A B C}\right) & =\min \left[\mathcal{S}(\rho \| \phi(\rho))-\mathcal{S}\left(\rho_{A} \| \phi_{A}\left(\rho_{A}\right)\right)\right. \\
& \left.-\mathcal{S}\left(\rho_{B} \| \phi_{B}\left(\rho_{B}\right)\right)-\mathcal{S}\left(\rho_{C} \| \phi_{C}\left(\rho_{C}\right)\right)\right] .
\end{aligned}
$$

For this specific tripartite state, we have:

$$
\begin{gathered}
\phi\left(\rho_{A B C}\right)=\sum_{k= \pm} \pi_{k} \rho_{A B C} \pi_{k}, \\
\pi_{k}=\pi_{A} \otimes \pi_{B} \otimes \pi_{c} .
\end{gathered}
$$

By tracing over the two subsystems B and C, $\rho_{A}$ is simply derived. Since $\rho_{A}$ is somehow a multiple of identity, therefore, $\mathcal{S}(\rho \| \phi(\rho))=0$. Similar expressions hold for $\rho_{A}$ and $\rho_{c}$. Hence, we could simply have

$$
\mathcal{D}\left(\rho_{A B C}\right)=\min _{\theta_{j}, \phi_{j}}[\mathcal{S}(\rho \| \phi(\rho))] .
$$

$\mathcal{S}(\rho)$ and $\mathcal{S}(\phi(\rho))$ are known by their eigenvalues as $-\operatorname{Tr}\left(\rho \log _{2} \rho\right)$; hence, they should be identified. As there are many parameters to be defined, we should like to make it simpler by considering the two cases of $\theta_{1}=0$ and $\phi_{j}=0$ [41]. The first term in $\rho_{A B C}$, Eq. (39), is a multiple of identity. It will, therefore, suffice to minimize the second part of the density matrix

$$
\mathcal{D}\left(\theta_{2}, \theta_{3}\right)=\min \left[-\sum_{j} \lambda_{j} \log \lambda_{j}\right]
$$

where, $\lambda_{j}$ s are the eigenvalues of $\mathcal{S}(\rho \| \phi(\rho))$ [41]

$$
\begin{aligned}
& \lambda_{1}=\lambda_{8}=\frac{1}{2} \cos ^{2}\left(\frac{\theta_{2}}{2}\right) \cos ^{2}\left(\frac{\theta_{3}}{2}\right), \\
& \lambda_{2}=\lambda_{7}=\frac{1}{2} \cos ^{2}\left(\frac{\theta_{2}}{2}\right) \sin ^{2}\left(\frac{\theta_{3}}{2}\right), \\
& \lambda_{3}=\lambda_{6}=\frac{1}{2} \sin ^{2}\left(\frac{\theta_{2}}{2}\right) \cos ^{2}\left(\frac{\theta_{3}}{2}\right), \\
& \lambda_{4}=\lambda_{5}=\frac{1}{2} \sin ^{2}\left(\frac{\theta_{2}}{2}\right) \sin ^{2}\left(\frac{\theta_{3}}{2}\right) .
\end{aligned}
$$

It is clearly seen that minimization occurs when $\theta_{2}=$ $\theta_{3}=0$. Now, the Expression for QD will take the follow- 


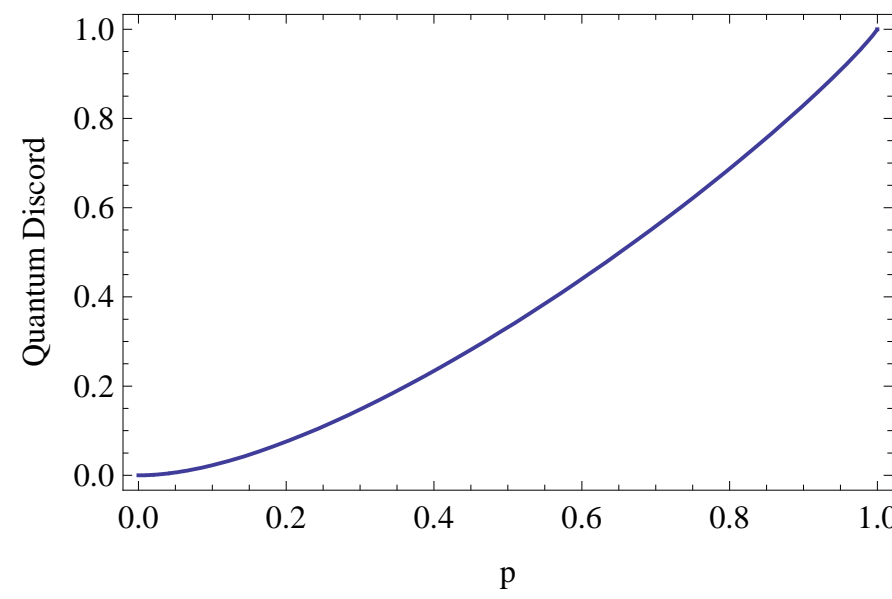

FIG. 4. Quantum Discord for a tripartite system versus p. Acceleration has no influence and this Figure exhibits a milder curvature than Fig. 2

ing form

$$
\begin{aligned}
\mathcal{D}\left(\rho_{A B C}\right) & =[\mathcal{S}(\rho \| \phi(\rho))]_{\theta_{j}=\phi_{j}=0} \\
& =7\left(\frac{1-p}{8}\right) \log _{2}\left(\frac{1-p}{8}\right)+\left(\frac{1+7 p}{8}\right) \log _{2}\left(\frac{1+7 p}{8}\right) \\
& -6\left(\frac{1-p}{8}\right) \log _{2}\left(\frac{1-p}{8}\right)-2\left(\frac{1+3 p}{8}\right) \log _{2}\left(\frac{1+3 p}{8}\right) \\
& =\frac{1}{8} \log _{2} \frac{(1+7 p)^{1+7 p}(1-p)^{1-p}}{(1+3 p)^{2(1+3 p)}} .
\end{aligned}
$$

Evidently, no effect of acceleration can be observed in this expression. The result is the same as that with the bipartite one: i.e., simply a function of p. This dependence on $\mathrm{p}$ is depicted in Fig. 4. Compared with the bipartite model (Fig. 2), it is seen that both figures are parabola but it is more curved in the bipartite system than it is in the tripartite one. Thus, we have shown another interesting feature of the particular helicity entangled state, which contradicts the degradation of QD in a non-inertial frame. In the next subsection, we would like to investigate another feature of this system which has come to be known as Geometric Quantum Discord.

\section{IV.3. Geometric Quantum Discord}

In studying Quantum information of the particular state introduced in this paper, we would like to investigate Geometric Quantum Discord as an alternative definition of quantum correlation. It is defined as the distance between the state being studied and the one with zero QD. This is known as the Hilbert-Schmidt distance [42]. In fact, Geometric QD is the square norm of the Hilbert-Schmidt distance; hence, it is called a 2-norm distance. Geometric QD could be quantitatively expressed as

$$
\mathcal{D}_{G}(\rho)=\min \left\|\rho-\rho^{\prime}\right\| .
$$

where, min is obtained over the measurements made. $\left\|\rho-\rho^{\prime}\right\|=\operatorname{Tr}\left(\left(\rho-\rho^{\prime}\right)^{2}\right)$ is the squared Hilbert-Schmidt norm. $\rho^{\prime}$ is the state with zero QD. This is the state in which the projective measurements $\pi_{i}$ have been made on some subsystems. This could be explicitly stated in the bipartite state as follows

$$
\rho^{\prime}=\sum_{i}\left(\pi_{i} \otimes 1\right) \rho\left(\pi_{i} \otimes 1\right),
$$

Finally, $\mathcal{D}_{G}(\rho)=\min _{\pi_{i}}|| \rho-\rho^{\prime} \|=\min _{\pi_{i}} \operatorname{Tr}\left(\left(\rho-\rho^{\prime}\right)^{2}\right)$.

It has been shown that Geometric QD is degraded in non-inertial frames [43]. Although the measure introduced here and the one introduced in the previous subsection are seemingly similar, they have certain basic differences. The latter goes to zero in the infinite limit acceleration [43] and its maximum value tends to 0.5 rather than 1 . We would like to investigate this feature in the density matrix given in this paper. Just similar to the case of QD, we would need to identify the projective measures. The measurements which are to be made effectively over a single qubit system are 43]

$$
\pi_{ \pm}=\frac{1}{2}(I \pm \vec{x} \cdot \vec{\sigma})
$$

where, $I$ is the identity, $\vec{x}=\left(x_{1}, x_{2}, x_{3}\right)$ is a unit vector $x_{1}^{2}+x_{2}^{2}+x_{3}^{2}=1$, and $\vec{\sigma}=\left(\sigma_{1}, \sigma_{2}, \sigma_{3}\right)$, and $\sigma$ s are pauli matrices. It has been clearly shown that minimization occurs when $x_{1}=x_{2}=0, x_{3}=1$ [44]. Accordingly, $\rho^{\prime}$ for a bipartite system will take the following form:

$$
\begin{aligned}
\rho^{\prime} & =\sum_{i= \pm}\left(\pi_{i} \otimes 1\right) \rho\left(\pi_{i} \otimes 1\right) \\
& =\left(1-e^{-2 \pi \omega}\right)^{2} \sum_{n=0}^{\infty} e^{-2 n \pi \omega}(n+1) \times \\
& \left(\frac { 1 - p } { 4 } \left(| 1 \rangle _ { + \uparrow A } | n + 1 \rangle _ { - \uparrow B } \left\langle1 | _ { + \uparrow A } \left\langlen+\left.1\right|_{-\uparrow B}\right.\right.\right.\right. \\
& +|1\rangle_{+\downarrow A}|n+1\rangle_{-\downarrow B}\left\langle\left. 1\right|_{+\downarrow A}\left\langle n+\left.1\right|_{-\downarrow B}\right)\right. \\
& +\frac{1+p}{4}\left(| 1 \rangle _ { + \uparrow A } | n + 1 \rangle _ { - \downarrow B } \left\langle1 | _ { + \uparrow A } \left\langlen+\left.1\right|_{-\downarrow B}\right.\right.\right. \\
& +|1\rangle_{+\downarrow A}|n+1\rangle_{-\uparrow B}\left\langle\left. 1\right|_{+\downarrow A}\left\langle n+\left.1\right|_{-\uparrow B}\right),\right.
\end{aligned}
$$

Using the basis $|1\rangle_{+\uparrow A}|n+1\rangle_{-\uparrow B}, \quad|1\rangle_{+\uparrow A} \mid n+$ $1\rangle_{-\downarrow B}, \quad|1\rangle_{+\downarrow A}|n+1\rangle_{-\uparrow B}, \quad|1\rangle_{+\downarrow A}|n+1\rangle_{-\downarrow B}$, (50) could be rewritten as follows

$$
\begin{array}{r}
\rho^{\prime}=\left(1-e^{-2 \pi \omega}\right)^{2} \sum_{n=0}^{\infty} e^{-2 n \pi \omega} \times \\
(n+1)\left(\begin{array}{cccc}
\frac{1-p}{4} & 0 & 0 & 0 \\
0 & \frac{1+p}{4} & 0 & 0 \\
0 & 0 & \frac{1+p}{4} & 0 \\
0 & 0 & 0 & \frac{1-p}{4}
\end{array}\right),
\end{array}
$$

which is the diagonal form of $\rho$. Now, Geometric QD, which is the trace norm of the difference between $\rho^{\prime}$ in Eq. (51) and $\rho$ from Eq. (27), is computed as in Eq. (52) below

$$
\mathcal{D}_{G}=\frac{p^{2}}{2}
$$




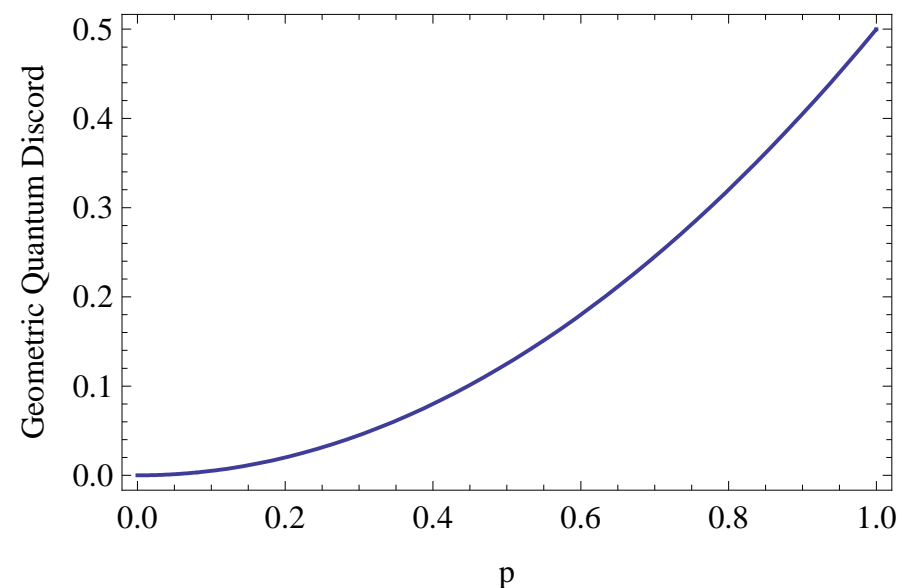

FIG. 5. Geometric Quantum Discord versus p. No dependence of acceleration is observed and it reaches its maximum value when $p=1$.

Geometric QD is depicted versus p in Fig. 5. Again, no effect of acceleration is seen and, as a consequence, it does not make any difference in the infinite acceleration limit. Also, its maximum value goes to 0.5 when $p=1$. This could also be generalized to systems higher than the bipartite one. For a general multipartite state, the global definition for Geometric QD is [45]

$$
\mathcal{D}_{G}=\min _{\left\{\pi_{a}\right\}}\left[\operatorname{Tr}\left(\rho^{2}\right)-\operatorname{Tr}\left(\rho^{\prime 2}\right)\right] .
$$

As an extension, we could find $\rho^{\prime}$ as a state with zero QD. It is very interesting to see that $\rho^{\prime}$ in this case is the diagonal form of $\rho$, too. Geometric QD would have the same expression as Eq.(52). Again, acceleration does not have any effect and the same properties hold as those for the bipartite case when $p=1$.

In addition, the Geometric QD as defined here has been shown to run into some difficulties under local operations upon the unmeasured subsystems [46]. A different definition is, therefore, introduced as the Geometric QD 1-norm which is the only possible p-norm. This is known as Schatten 1-norm and is able to consistently quantify non-classical correlations [47]. It is defined as $\mathcal{D}_{G}=\min \operatorname{Tr}\left[\rho-\rho^{\prime}\right]$, where $\rho, \rho^{\prime}$ are identified before. This is not in the interest of this paper but it could be calculated and for the specific case used here is equal to $p$.

\section{BEYOND SINGLE MODE ANALYSIS}

Up until now, the results given are in the limit of the single mode approximation. It is interesting to study whether or not the entangled state introduced here preserves its specific features beyond the single mode approximation. Generally, the single mode approximation is not valid for all states. It only holds for some wavepackets related to the Minkowski ones if fourier transforms are imposed. In this analysis, the entangled state is quantified between a Minkowski and a Unruh mode instead of the Rindler mode. This special mode is defined by its special base and could be written as a linear combination of the Rindler bases as follows [48]

$$
\begin{aligned}
& u_{\Omega, R}=\cosh \left(r_{\Omega}\right) u_{\Omega, I}+\sinh \left(r_{\Omega}\right) u_{\Omega, I I}^{*}, \\
& u_{\Omega, L}=\cosh \left(r_{\Omega}\right) u_{\Omega, I I}+\sinh \left(r_{\Omega}\right) u_{\Omega, I}^{*} .
\end{aligned}
$$

In this equation, $u_{\Omega, R(L)}$ 's are the Unruh right (left) bases and $u_{\Omega, I(I I)}$ 's are the first (second) Rindler bases, also $\tanh \left(r_{\Omega}\right)=\exp (-\pi \Omega)$. Their corresponding creation and annihilation operator could be performed in a similar manner. Since the transformations between Minkowski and Unruh modes do not mix, they could have the same vacuum state [24]

$$
\begin{aligned}
\left|0_{M}\right\rangle & =\left|0_{\Omega}\right\rangle=\prod_{\Omega}\left|0_{\Omega}\right\rangle_{U}, \\
\left|0_{\Omega}\right\rangle_{U} & =\sum_{n} \frac{\tanh \left(r_{\Omega}\right)^{n}}{\cosh \left(r_{\Omega}\right)}\left|n_{\Omega}\right\rangle_{I}\left|n_{\Omega}\right\rangle_{I I} .
\end{aligned}
$$

The other number states could be formed by implying the creation Unruh operator on the vacuum state [24]

$$
\begin{aligned}
a_{\Omega, U}^{\dagger}\left|0_{\Omega}\right\rangle_{U} & =\sum_{n=0}^{\infty} \frac{\tanh \left(r_{\Omega}\right)^{n}}{\cosh \left(r_{\Omega}\right)} \frac{\sqrt{n+1}}{\cosh \left(r_{\Omega}\right)}\left|\phi_{\Omega}^{n}\right\rangle \\
\left|\phi_{\Omega}^{n}\right\rangle & =q_{L}\left|n_{\Omega}\right\rangle_{I}\left|n+1_{\Omega}\right\rangle_{I I}+q_{R}\left|n+1_{\Omega}\right\rangle_{I}\left|n_{\Omega}\right\rangle_{I I} .
\end{aligned}
$$

where, $\left|q_{R}\right|^{2}+\left|q_{L}\right|^{2}=1$. The special case $q_{R}=1, q_{L}=0$ indicates the single mode approximation. Clearly, this special choice breaks the symmetry between right and left wedges. For the bipartite entangled state considered in this paper Eq.(26), we need to define the state $\left|1_{\Omega}\right\rangle_{+\uparrow U}$ along the following lines:

$$
\begin{gathered}
\left|1_{\Omega}\right\rangle_{+\uparrow U}=\left(1-e^{-2 \pi \Omega}\right) \sum_{n=0}^{\infty} e^{-2 n \pi \Omega} \sqrt{(n+1)}(57) \\
\left(q_{L}\left|n_{\Omega}\right\rangle_{+\uparrow}^{I}\left|(n+1)_{\Omega}\right\rangle_{-\uparrow}^{I I}+q_{R}\left|(n+1)_{\Omega}\right\rangle_{+\uparrow}^{I}\left|n_{\Omega}\right\rangle_{-\uparrow}^{I I}\right) .
\end{gathered}
$$

As mentioned above, the density matrix should be computed for quantifying QE. By tracing over the second region, the result would be obtained as the Alice-Bob density matrix; namely, 


$$
\begin{aligned}
& \rho_{A B}=\frac{1}{2}\left(1-e^{-2 \pi \Omega}\right)^{2} \sum_{n=0}^{\infty} e^{-2 n \pi \Omega}(n+1) \times \\
& \quad\left(| q _ { L } | ^ { 2 } \left(| 1 \rangle _ { + \uparrow A } | n \rangle _ { - \downarrow B } \langle 1 | _ { + \uparrow A } \langle n | _ { - \downarrow B } + | 1 \rangle _ { + \downarrow A } | n \rangle _ { - \downarrow B } \left\langle1 | _ { + \uparrow A } \left\langle\left.n\right|_{-\uparrow B}\right.\right.\right.\right. \\
& +|1\rangle_{+\uparrow A}|n\rangle_{-\uparrow B}\left\langle\left. 1\right|_{+\downarrow A}\left\langle\left. n\right|_{-\downarrow B}+\mid 1\right\rangle_{+\downarrow A} \mid n\right\rangle_{-\uparrow B}\left\langle\left. 1\right|_{+\downarrow A}\left\langle\left. n\right|_{-\uparrow B}\right)\right. \\
& +\left(| q _ { R } | ^ { 2 } \left(| 1 \rangle _ { + \uparrow A } | n + 1 \rangle _ { - \downarrow B } \langle 1 | _ { + \uparrow A } \langle n + 1 | _ { - \downarrow B } + | 1 \rangle _ { + \downarrow A } | n + 1 \rangle _ { - \downarrow B } \left\langle1 | _ { + \uparrow A } \left\langlen+\left.1\right|_{-\uparrow B}\right.\right.\right.\right. \\
& +|1\rangle_{+\uparrow A}|n+1\rangle_{-\uparrow B}\left\langle\left. 1\right|_{+\downarrow A}\left\langle n+\left.1\right|_{-\downarrow B}+\mid 1\right\rangle_{+\downarrow A} \mid n+1\right\rangle_{-\uparrow B}\left\langle\left. 1\right|_{+\downarrow A}\left\langle n+\left.1\right|_{-\uparrow B}\right)\right) .
\end{aligned}
$$

In the process of studying Negativity, the partial transpose of the density matrix given in Eq. (58) should be taken. The related eigenvalues are

$$
\begin{gathered}
\frac{1}{2}\left(1-e^{-2 \pi \Omega}\right)^{2} \sum_{n=0}^{\infty} e^{-2 n \pi \Omega}\left\{\left|q_{R}\right|^{2},\left|q_{R}\right|^{2},\left|q_{R}\right|^{2}\right. \\
\left.-\left|q_{R}\right|^{2},\left|q_{L}\right|^{2},\left|q_{L}\right|^{2},\left|q_{L}\right|^{2},-\left|q_{L}\right|^{2}\right\}
\end{gathered}
$$

Therefore, Negativity is expressed by:

$$
\log _{2} \frac{1}{2}\left(1-e^{-2 \pi \Omega}\right)^{2} \sum_{n=0}^{\infty} e^{-2 n \pi \Omega}\left(4\left(\left|q_{R}\right|^{2}+\left|q_{L}\right|^{2}\right)\right)=1
$$

It is clearly seen that acceleration dependency disappears. Beyond the single mode approximation, we again find $\mathrm{QE}$ to be observer independent. It is interesting that the dependence of $q_{R}$ and $q_{L}$ also disappeared. By exchanging $q_{R}$ by $q_{L}$, the Alice-AntiBob density matrix could be easily derived. The same result is obtained for the Alice-AntiBob system. Going further, we would like to investigate QD beyond the single mode approximation. The approach adopted for calculating QD was introduced in Section 4. Defining the density matrix as in Eq.(26), using Eq.(7) as $|\psi\rangle$, and by tracing over the second (first) region, the density matrix Eq.(25) for Alice-(Anti)Bob is derived. QD for this special state beyond the single mode approximation is obtained as follows

$$
\begin{aligned}
D(A ; B) & =\frac{1+3 p}{4}\left|q_{L}\right|^{2} \log _{2}\left(\frac{1+3 p}{4}\left|q_{L}\right|^{2}\right)+\frac{1-p}{4}\left|q_{L}\right|^{2} \log _{2}\left(\frac{1-p}{4}\left|q_{L}\right|^{2}\right) \\
& +\frac{1+3 p}{4}\left|q_{R}\right|^{2} \log _{2}\left(\frac{1+3 p}{4}\left|q_{R}\right|^{2}\right)+\frac{1-p}{4}\left|q_{R}\right|^{2} \log _{2}\left(\frac{1-p}{4}\left|q_{R}\right|^{2}\right) \\
& -\frac{1+3 p}{4}\left|q_{L}\right|^{2} \log _{2}\left(\frac{1+p}{4}\left|q_{L}\right|^{2}\right)-\frac{1-p}{4}\left|q_{L}\right|^{2} \log _{2}\left(\frac{1+p}{4}\left|q_{L}\right|^{2}\right) \\
& -\frac{1+3 p}{4}\left|q_{R}\right|^{2} \log _{2}\left(\frac{1+p}{4}\left|q_{R}\right|^{2}\right)-\frac{1-p}{4}\left|q_{R}\right|^{2} \log _{2}\left(\frac{1+p}{4}\left|q_{R}\right|^{2}\right) .
\end{aligned}
$$

Here, QD only depends on $q_{R}, q_{L}$, and p. Using the expression $\left|q_{R}\right|^{2}+\left|q_{L}\right|^{2}=1$, the above equation could be further simplified to

$$
\begin{aligned}
D(A ; B) & =\frac{(1-p) \log _{2}(1-p)-2(1+p) \log _{2}(1+p)}{\log _{2} 16} \\
& +\frac{(1+3 p) \log _{2}(1+3 p)}{\log _{2} 16} \\
& =\frac{1}{4} \log _{2} \frac{(1+3 p)^{1+3 p}(1-p)^{1-p}}{(1+p)^{2(1+p)}}
\end{aligned}
$$

Therefore, it is independent of different values of $q_{R}$ and $q_{L}$. Evidently, it is only p-dependent. If we factorize this expression, we would exactly have Eq. (36), which is acceleration independent. It is observed that not only does increment of acceleration have no impact on the Quantum features for this specific helicity entangled state, but neither does extending our approximation to beyond the single mode make any difference to the value of Quantum Discord and Quantum Entanglement. This is another interesting peculiarity of this helicity entangled state which makes it a specific state in Quantum Information Theory. Some other quantum features such as Geometric Quantum Discord could be computed in this limit. The tripartite helicity entangled state could also be a good candidate for investigating QE and QD in the limit beyond the single mode approximation.

\section{SUMMARY AND CONCLUSIONS}

In summary, we investigated some quantum features of a specific state which is entangled in the helicity part. It has been shown that degradation of Quantum Entanglement for this special case cancels when acceleration increases. Here, we extended this feature to multipartite states. Particularly, we showed that a tripartite entangled state yields the same result and the degree of the entangled state remains unchanged. Moreover, Quantum Discord as a measure of quantum correlation was studied for the special bipartite and tripartite systems. A general definition was given for multipartite systems. 
Using this global definition, QD was computed for a specific tripartite system. Acceleration was found to have no effect on this feature, either. Another property investigated in the present paper was the special distance which tends toward zero in the infinite acceleration limit. For the state considered here, however, acceleration was found to disappear for the Geometric Quantum Discord 2-norm. This last measure was seen to have potential problems under local operations upon the unmeasured subsystems [46]. The definition could have been possibly modified by considering 1-norm of this distance, but it was not of interest in this paper. These quantum features have been studied in the analysis of beyond the single mode. A basic result which makes this state an important state is that beyond the single mode approximation, Negativity, as a measure of QE, remains constant in the value of a maximally entangled. Here, we have also computed $\mathrm{QD}$ and noticed that this quantity which defines the degree of a quantum correlation also remains unchanged. By computing these quantities, it is observed that quantum correlations for this helicity entangled state is preserved in non-inertial frames in both the single and beyond the single mode approximation. Investigating QE, QD, and Geometric Quantum Discord for the specific tripartite helicity entangled state which was introduced here is suggested for further research in the limit of beyond the single mode approximation.
[1] M. A. Nielsen and I. L. Chuang, Quantum Computation and Quantum Information, Cambridge University Press, 2000.

[2] A. K. Ekert, Phys. Rev. Lett. 67, 661 (1991).

[3] C. H. Bennett, G. Brassard and N. D. Mermin, Phys. Rev. Lett. 68, 557 (1992).

[4] C. H. Bennett, G. Brassard, C. Crepeau, R. Jozsa, A. Peres and W. K. Wootters, Phys. Rev. Lett. 70, 1895 (1993).

[5] R. P. Feynman, Int. J. Theor. Phys. 21, 467 (1982).

[6] P. Shor, in Proc. 35th Annual Symp. Foundations Comp. Sci. 124 (IEEE Press, 1994).

[7] C. H. Bennett, and S. J. Wiesner, Phys. Rev. Lett. 69, 2881(1992).

[8] K. Modi, A. Brodutch, H. Cable, T. Paterek, and V. Vedral, Rev. Mod. Phys. 84, 1655 (2012).

[9] L. Henderson and V. Vedral, J. Phys. A 34, 6899 (2001).

[10] H. Ollivier and W. H. Zurek, Phys. Rev. Lett. 88, 017901 (2001).

[11] Lafamme, R., D. G. Cory, C. Negrevergne, and L. Viola, Quantum Inf. Comput. 2, 1662002.

[12] Datta, A., Studies on the Role of Entanglement in Mixedstate Quantum Computation, Ph.D. thesis, Univer-sity of New Mexico, arXiv:0807.4490 [quant-ph] June 2008.

[13] L. Bombelli, R. K. Koul, J. Lee, and R. Sorkin, Phys. Rev. D 34, 373 (1986).

[14] S. W. Hawking, Commun. Math. Phys. 43, 199 (1975); Phys. Rev. D 14, 2460 (1976); H.Terashima, Phys. Rev. D 61, 104016 (2000).

[15] G. Adesso, I. Fuentes-Schuller, and M. Ericsson, Phys. Rev. A 76, 062112 (2007).

[16] R. B. Mann and V. M. Villalba, Phys. Rev. A 80, 022305 (2009).

[17] P. M. Alsing and I. Fuentes, Class. Quantum Grav. 29, (2012).

[18] P. M. Alsing, and G. J. Milburn Phys. Rev. Lett. 91 180404 (2003).

[19] I. Fuentes-Schuller, and R. B. Mann, Phys. Rev. Lett. 95, 120404 (2005).

[20] S. Khan, Ann. Phys. DOI: 10.1016/j.aop.2014.05.022 (2014).

[21] H. Mehri-Dehnavi, B. Mirza, H. Mohammadzadeh, and R. Rahimi, Ann. of Phys. 326, 1320 (2011).

[22] Y. Ling, S. He, W. Qiu and H. Zhang, J. Phys. A: Math. Theor. 40, 9025 (2007).
[23] S. A. A. Ghorashi, M. H. Aminjavaheri, M. Bagheri Harouni, Quant. Inf. Process. DOI 10.1007/s11128-0130668-8 (2013).

[24] D. E. Bruschi, J. Louko, E. Martin-Martinez, A. Dragan, and I. Fuentes, Phys. Rev. A 82, 042332 (2010).

[25] D. E. Bruschi, A. Dragan, I. Fuentes, J. Louko, Phys. Rev. D 86, 025026 (2012).

[26] E. Martin-Martinez, D. Hosler, M. Montero, Phys. Rev. A 86, 062307 (2012).

[27] N. Friis, P. Khler, E. Martin-Martinez, R. A. Bertlmann, Phys. Rev. A 84, 062111 (2011).

[28] M. Ramzan, Chin. Phys. Lett. 30, 060307 (2013).

[29] W. G. Unruh, and R. Schutzhold, Phys. Rev. D 71, 034028 (2005).

[30] S. Carroll, Spacetime and Geometry: An Introduction to General Relativity, Benjamin Cummings Publishers, 2003.

[31] P. M. Alsing, I. Fuentes-Schuller, R. B. Mann, and T. E. Tessier, Phys. Rev. A 74, 032326 (2006).

[32] D. Bruss, J. Math. Phys. 43, 4237 (2002);

G. Vidal and R. F. Werner, Phys. Rev. A 65, 032314 (2002).

[33] S. M. Barnett, Quantum Information, Oxford University Press, 2009.

[34] A. Peres, Quantum theory: concepts and methods Kluwer Academic Publishers, Dordrecht, 1995.

[35] J. Wang, and J. Jing, Phys. Rev. A 83, 022314 (2011).

[36] Y. U. Ou and H. Fan, Phys. Rev. A 75, 062308 (2007).

[37] V. Coffman, J. Kundu, and W. K. Wootters, Phys. Rev. A 61, 052306 (2000).

[38] T.M. Cover, J.A. Thomas, Elements of Information Theory, Ed. J. Wiley (1991).

[39] M. Ali, A. R. P. Rau, and G. Alber, Phys. Rev. A 81, 042105 (2010).

[40] S. Luo, Phys. Rev. A 77, 042303 (2008).

[41] C. C. Rulli, and M. S. Sarandy, Phys. Rev. A 84, 042109 (2011)

[42] B. Dakic, V. Vedral and C. Brukner, Phys. Rev. Lett. 105, 190502 (2010);

S. Luo and S. Fu, Phys. Rev. A 82, 034302 (2010).

[43] E. G. Brown, K. Cormier, E. Martin-Martinez, and R. B. Mann, Phys. Rev. A 86, 032108 (2012).

[44] A. Datta, A. Shaji, and C. M. Caves, Phys. Rev. Lett. 100, 050502 (2008).

[45] J. Xu, J. Phys. A: Math. Theor. 45, 405304 (2012).

[46] M. Piani, arXiv:1206.0231v2 [quant-ph] 25 July 2012. 
[47] F. M. Paula, T. R. de Oliveira, and M. S. Sarandy, arXiv:1302.7034v1 [quant-ph] 27 Feb 2013.

[48] N. D. Birrell and P. C. W. Davies, Quantum Fields in Curved Space (Cambridge University Press, 1984). 